\title{
Nicotine is Insufficient as a Carcinogen, It's Functions as a Tumor Promoter on Purpose
}

\author{
Jia Ping Wu* \\ National Taipei University of Nursing and Health Sciences, Taiwan
}

*Corresponding author: Jia Ping Wu, Research Center for Healthcare Industry Innovation, National Taipei University of Nursing and Health Sciences, Taipei City, Taiwan.

To Cite This Article: Jia Ping Wu. Nicotine is Insufficient as a Carcinogen, It's Functions as a Tumor Promoter on Purpose. Am J Biomed Sci \& Res. 2019 - 5(1). AJBSR.MS.ID.000872. DOI: 10.34297/AJBSR.2019.05.000872

Received: 眥 August 29, 2019; Published: 眥 September 04, 2019

\begin{abstract}
Nicotine constitutes approximately $0.6 \sim 3.0 \%$ of the dry weight of tobacco. Like anything that enters the body, nicotine is also metabolized. Therefore, any activity that increases your metabolic rate can help speed up the clearance of nicotine. Nicotine also promotes cancer growth, angiogenesis, and neovascularization. Thereby, nicotine impeding apoptosis, promoting tumor growth and activating growth factors. This article provides a brief description of nicotine is unusual in comparison to most drugs, as its concentration profile changes from induced tumor growth to cytotoxic with increasing doses. The concentration of nicotine stimulated cell growth correspond to low concentration was needed, while high concentration was cytotoxic.
\end{abstract}

Keywords: Carcinogen; Nicotine; Tumor growth; Cytotoxic; Apoptosis

\section{Introduction}

Tobacco smoking lead to DNA damage. Cigarette smoke contains more than 6,000 components [1]. Smoking is the leading risk factor for lung cancer. Secondhand smoke exposure or air pollution appears to be the primary underlying cause of cancer. Exercise is a good way to increase the rate of metabolism. Exercise improves heart rate and increases the rate of metabolism and burning of heat [2]. For people who have many years of smoking, it is important to start exercising. Make sure to drink plenty of water because nicotine is soluble in water, so drinking water helps to excrete the substance through the urine. Vitamin A is also helpful in removing nicotine from the body because it also has the effect of speeding up the metabolism [3]. Because nicotine tends to destroy vitamin C in the body, it is important to supplement it after quitting smoking. Nicotine is highly addictive. It follows that nicotine is associated with cancer in humans [4]. Therefore, effects on chemotherapeutics by several malignant cell lines, nicotine in concentrations as low as $1 \mu \mathrm{M}$ decreased. Because of treatment with $1 \mu \mathrm{M}$ nicotine significantly enhanced the frequency of formation of micronuclei, although a higher nicotine concentration. Nicotine acts as tumor growth promoted resistance to apoptosis leading to carcinogenesis [5]. Nicotine is concomitant dual effects on anti-apoptosis and genotoxic activity.

Nicotine is unusual in comparison to most drugs. With increasing dosages, its profile changes from stimulant to sedative. At very high doses it dampens neuronal activity [6]. This phenomenon is as well-known "Nesbitt's paradox". Micronuclei are characterized in the cancerous cells have some sort of DNA damage. Micronuclei body is a small body can be seen in a newly divided daughter cell. Micronuclei body increased is usually an indication of increased DNA damage or mutation [7]. The mechanisms leading to the formation of Micronuclei body are chromosome breakage and disturbance of the chromosome-segregation system, which represents an irreversible DNA damage. This mechanism responsible for the genotoxic effects caused by nicotine [8]. Effects of nicotine on angiogenesis have been demonstrated for lung tumor cells. Reports had also been demonstrated in H157 lung cancer, where nicotine significantly increased the size and number of tumors in the lung [9]. On the other hand, at high nicotine concentrations $(>1 \mu \mathrm{M})$ with consistent cytotoxic effects and appeared to be due to direct cell kill. Cellular cytotoxicity was associated with inhibition of DNA synthesis, not stimulation of DNA synthesis. This is the main way that micronuclei are formed [10]. Micronuclei can also be spontaneously formed as a byproduct of inhibition of DNA synthesis. This mechanism to micronuclei formation is by a double-strand break DNA, creating a separate linear fragment lead to formation of a micronucleus. Micronuclei are small [11].

These extranuclear bodies that are formed during mitosis from lagging chromosomes. This results in parts of the cell senses extra chromosomes, the cell can attempt to remove the extra chromo- 
somes in another cell membrane, separate from the other normal chromosomes being broken off and enveloped as an extra nucleus in one of the daughter cells [12]. Nicotine is an important component in tobacco. Among various subtypes of nicotinic receptors, homopentamers of $\alpha 7 \mathrm{nAChR}$ can bind nicotine with highest affinity and mediate multiple effects of nicotine in lung cancer [13]. nAChRs expressed on lung carcinoma or mesothelioma form a part of an autocrine-proliferative network facilitating the growth of neoplastic cells. Target drugs as a form of molecular medicine, targeted therapy blocks the growth of cancer cells by interfering with specific targeted molecules needed for carcinogenesis and tumor growth $[14,15]$. Nicotine could induce the proliferation of a variety of lung carcinoma cell clines, but there is no evidence that nicotine itself provokes cancer. Nicotine alone is generally accepted as a tumor promoter, but not a tumor initiator in carcinogenesis [16,17]. Nicotine can prevent apoptosis induced by various agents in NSCLC.

\section{Conclusion}

The concentrations of nicotine promote cell proliferation correspond to the low concentrations, while high concentrations are cytotoxic. During nicotine in concentrations as low as $1 \mu \mathrm{M}$, nicotine activates cell migration, proliferation, survival, and anti-apoptotic effects exerted, in contract, modulation chemotherapeutics on several different malignant cell lines. This phenomenon which nicotine-mediated inhibition of apoptosis may contribute to observed in normal and transformed cells derived from the pathogenesis of tobacco use-related cancer use as well as decrease the efficacy of cancer therapies.

\section{Acknowledgement}

This work was supported by grants from Ministry of Science and Technology (MOST 105-2811-B-039-008 and MOST 106-2811B-650-003).

\section{Conflict of interest}

The authors do not have any conflict of interest in the manuscript.

\section{References}

1. Zhang J, Kamdar O, Le W, Rosen GD, Upadhyay D (2009) Nicotine induces resistance to chemotherapy by modulating mitochondrial signaling in lung cancer. Am J Respir Cell Mol Biol 40(2): 135-146.

2. Grozio A, Catassi A, Cavalieri Z, Paleari L, Cesario A, et al. (2007) Nicotine, lung and cancer. Anticancer Agents Med Chem 7(4): 461-466.
3. Jensen KP, DeVito EE, Sofuoglu M (2016) How Intravenous Nicotine Administration in Smokers Can Inform Tobacco Regulatory Science. Tob Regul Sci 2(4): 452-463.

4. Warren GW, Singh AK (2013) Nicotine and lung cancer. J Carcinog 12: 1.

5. Bordas A, Cedillo JL, Arnalich F, Esteban Rodriguez I, Guerra Pastrián L, et al. (2017) Expression patterns for nicotinic acetylcholine receptor subunit genes in smoking-related lung cancers. Oncotarget 8(40): 67878-67890.

6. Xu J, Huang H, Pan C, Zhang B, Liu X, et al. (2007) Nicotine inhibits apoptosis induced by cisplatin in human oral cancer cells. Int J Oral Maxillofac Surg 36(8): 739-744.

7. Fan Y, Wang K (2017) Nicotine induces EP4 receptor expression in lung carcinoma cells by acting on AP- $2 \alpha$ : The intersection between cholinergic and prostanoid signaling. Oncotarget 8(44): 75854-75863.

8. Yan YE, Liu L, Wang JF, Liu F, Li XH, et al. (2014) Prenatal nicotinic exposure suppresses fetal adrenal steroidogenesis via steroidogenic factor 1 (SF-1) deacetylation. Toxicol Appl Pharmacol 277(3): 231-241.

9. Grill S, Yahiaoui Doktor M, Dukatz R, Lammert J, Ullrich M, et al. (2017) Smoking and physical inactivity increase cancer prevalence in BRCA-1 and BRCA-2 mutation carriers: results from a retrospective observational analysis. Arch Gynecol Obstet 296(6): 1135-1144.

10. Togashi Y, Hayashi H, Okamoto K, Fumita S, Terashima M, et al. (2015) Chronic nicotine exposure mediates resistance to EGFR-TKI in EGFR-mutated lung cancer via an EGFR signal. Lung Cancer 88(1): 16-23.

11. Villablanca AC (1998) Nicotine stimulates DNA synthesis and proliferation in vascular endothelial cells in vitro. J Appl Physiol (1985) 84(6): 2089-2098.

12. Dutra Tavares AC, Silva JO, Nunes Freitas AL, Guimarães VMS, Araújo UC, et al. (2017) Maternal undernutrition during lactation alters nicotine reward and DOPAC/dopamine ratio in cerebral cortex in adolescent mice, but does not affect nicotine-induced nAChRs upregulation. Int J Dev Neurosci 65: 45-53.

13. Khezri S, Abtahi Froushani SM, Shahmoradi M (2018) Nicotine Augments the Beneficial Effects of Mesenchymal Stem Cell-based Therapy in Rat Model of Multiple Sclerosis. Immunol Invest 47(2): 113-124.

14. Wright SC, Zhong J, Zheng H, Larrick JW (1993) Nicotine inhibition of apoptosis suggests a role in tumor promotion. FASEB J 7(11): 10451051.

15. Jia Y, Sun H, Wu H, Zhang H, Zhang X, et al. (2016) Nicotine Inhibits Cisplatin-Induced Apoptosis via Regulating $\alpha 5$-nAChR/AKT Signaling in Human Gastric Cancer Cells. PLoS ONE 11(2): e0149120.

16. Alex I Chernyavsky, Igor B Shchepotin, Valentin Galitovkiy, Sergei A Grando (2015) Mechanisms of tumor-promoting activities of nicotine in lung cancer: synergistic effects of cell membrane and mitochondrial nicotinic acetylcholine receptors. BMC Cancer 15: 152

17. Rebecca E Schane, Pamela M Ling, Stanton A Glantz (2010) Health Effects of Light and Intermittent Smoking. Circulation 121: 1518-1522. 